\title{
Plädoyer für eine operationalisierbare Methode der Prozesserhebung in der Beratung
}

\section{Klassifikation bestehender Ansätze und Implikationen für die Beratungspraxis}

\author{
Silke Balzert, Peter Fettke, Peter Loos \\ Institut für Wirtschaftsinformatik \\ im Deutschen Forschungszentrum für Künstliche Intelligenz, \\ Universität des Saarlandes
}

\section{Motivation und Problemstellung}

Mit einem Umsatz von 18,2 Milliarden Euro und mehr als 115.000 Beschäftigen (Stand: 2008) stellt die Beratungsbranche einen bedeutenden Wirtschaftszweig in Deutschland dar. Gleichwohl steht die wissenschaftliche Auseinandersetzung mit dem Thema Unternehmensberatung erst am Anfang. So wird aufgrund der weitgehenden Isolation der bisherigen Forschungsbemühungen in der Literatur die Institutionalisierung eines entsprechenden Forschungsfeldes Consulting Research gefordert (Nissen 2007, S. 14; sowie die dort genannten Quellen), um sowohl die wissenschaftliche Durchdringung des Themas Unternehmensberatung zu forcieren als auch wissenschaftliche Methoden und Erkenntnisse in die Beratungspraxis zu transferieren.

Der Ergebnistransfer in die Praxis ist auch Gegenstand des vorliegenden Beitrags, wobei aus zwei Gründen speziell das Geschäftsprozessmanagement und die Geschäftsprozessreorganisation fokussiert werden. Zum einen entfallen in Deutschland rund 8 Milliarden Euro Umsatz allein auf den Bereich der Organisations- und Prozessberatung (BDU 2009, S. 3) und Untersuchungen zeigen, dass dieser Markt für Beratungsunternehmen hoch lukrativ ist (Hess und Schuller 2005, S. 370-371). Zum anderen scheint sich in der Literatur bisher kein Vorgehensmodell zur Prozessmodellierung im Allgemeinen und zur Prozesserhebung im Speziellen etabliert zu haben, welches allgemein akzeptiert wird (Eggert 2009, S. 2; sowie die dort zitierte Quelle), und auch zur Geschäftsprozessreorganisation hat sich offenbar noch keine standardisierte Methode durchgesetzt (Hess und Schuller, 2005, S. 355-373). 
Diese Diskrepanz zwischen hoher wirtschaftlicher Bedeutung einerseits und mangelhafter methodischer Durchdringung andererseits ist zunächst irritierend, da Hammer und Champy bereits Anfang der 1990er Jahre die radikale Methode des Business Process Reengineering vorschlugen (Hammer und Champy 1994). In der darauf folgenden Diskussion des Themas in Wissenschaft und Praxis setzte sich die Erkenntnis durch, dass der wirtschaftliche Erfolg eines Unternehmens maßgeblich auf effiziente Geschäftsprozesse zurück geführt werden kann (Jost und Kruppke 2004). Entsprechend wäre nach dieser langen Zeitspanne eine tiefgehende Durchdringung der Thematik in Wissenschaft und Praxis zu erwarten.

Ziel des vorliegenden Beitrags ist es, diesen Durchdringungsgrad anhand einer Auswahl von etablierten Lehrbüchern zum Geschäftsprozessmanagement zu untersuchen und daraus Implikationen für die Beratungspraxis abzuleiten. Der Betrachtungsschwerpunkt liegt dabei auf der Phase der Prozesserbebung, da ihr eine hohe Bedeutung zur Identifikation von Schwachstellen und Verbesserungspotenzialen zugesprochen wird (Schwegmann und Laske 2005, S. 155-157). Ferner ist eine solche Prozesserhebung meist mit erheblichem Aufwand verbunden und kann daher in besonderem Maße von einer strukturierten und effizienten Vorgehensweise profitieren, die theoretisch fundiert und auch in die Praxis umsetzbar - also operationalisierbar - ist. Im vorliegenden Beitrag soll deshalb insbesondere untersucht werden, welche derartigen Vorgehensmodelle zur Prozesserhebung in der Literatur existieren und wie gut sie in der Praxis ein- und umsetzbar sind.

Um den Beitrag thematisch zu verorten wird zunächst in Abschnitt 2 der terminologische Bezugsrahmen dargelegt, bevor in Abschnitt 3 die untersuchten Literaturquellen inhaltlich dargestellt und entsprechend der definierten Fragestellung kritisch gewürdigt werden. Anschließend wird in Abschnitt 4 ein Klassifikationsschema zur Einordnung der verschiedenen Vorgehensmodelle vorgestellt. Der Beitrag schließt mit einer Zusammenfassung der wichtigsten Ergebnisse und den daraus resultierenden Implikationen für die Beratungspraxis sowie einem Ausblick.

\section{Terminologischer Bezugsrahmen}

In diesem Abschnitt werden zunächst die grundlegenden Begriffe eingeführt und der Beitrag so thematisch verortet. Gegenstand der Prozesserhebung sind Geschäftsprozesse, d. h. „zusammengehörende Abfolgen von Unternehmensverrichtungen zum Zweck einer Leistungserstellung" (Scheer 2002, S. 3). Ziel der Prozesserhebung ist die Erstellung von Prozessmodellen, die eine Teilmenge aller Modelle darstellen.

Zur Definition des Begriffs Modell existieren in der Wirtschaftsinformatik mehrere Ansätze, die ein Modell über seine Funktion bzw. seine Entstehung charakterisieren (für den abbildungsorientierten bzw. konstruktionsorientierten Modellbegriff vgl. exemplarisch Schütte (1998, S. 59) und vom Brocke (2003, S. 10-12)). Vom Brocke differenziert in seinem konstruktionsprozessorientierten Modellbegriff den Prozess der Modellerstellung und interpretiert diese als eine Folge von Zuständen, die das 
zu entwickelnde Modell einnimmt (vom Brocke 2003, S. 15-20). Wie in Abbildung 1 dargestellt, geht der Modellkonstrukteur bei seiner Arbeit von einem Ausgangsmodell aus, das die Basis für sein eigenes mentales Modell bildet. In intersubjektiver Abstimmung mit dem Modellnutzer erweitert und verändert er dieses interne Modell so lange (Modellzustände), bis mit dem sog. Ergebnismodell der Endzustand, d.h. ein abgestimmtes und expliziertes Modell erreicht ist, dessen Entwicklung immer einem definierten Modellzweck dient, den der Modellnutzer vorgibt.

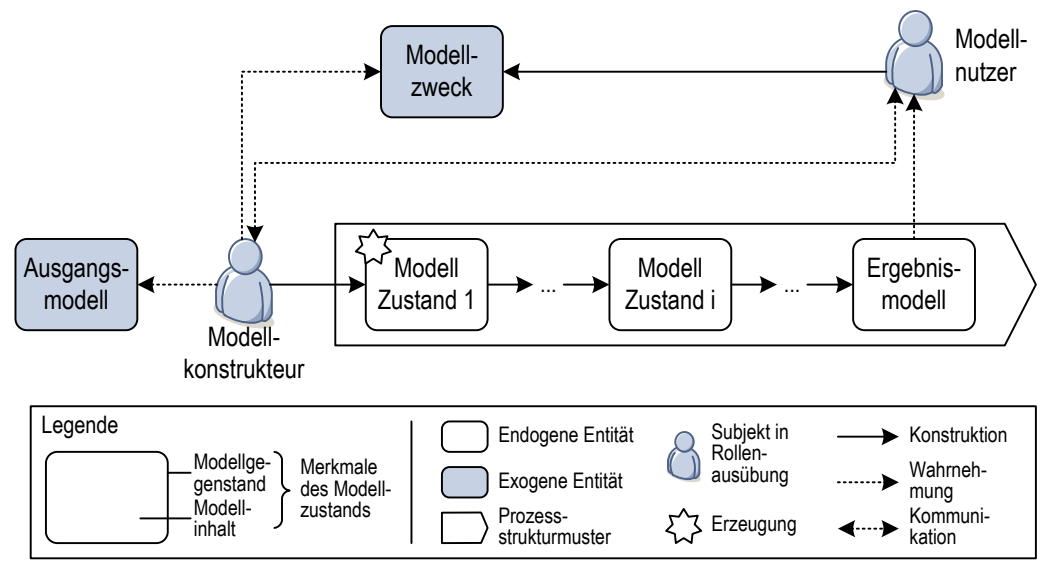

Abbildung 1: Konstruktionsprozessorientierter Modellbegriff nach vom Brocke

Diesem konstruktionsprozessorientierten Modellbegriff folgend umfasst der Begriff der Prozesserbebung in diesem Beitrag alle notwendigen Schritte, die zur Bildung des Ausgangs- und Ergebnismodells notwendig sind. Er untersucht daher, welche Schritte in der Literatur als notwendig erachtet werden, um eine Prozesserhebung von der Bildung des Ausgangsmodells bis zum explizierten Ergebnismodell durchzuführen, und in welchem Maße die Literatur Hinweise zur Operationalisierung dieser Schritte gibt. Dabei wird unterstellt, dass die Rolle des Modellkonstrukteurs von einem Unternehmensberater und die Rolle des Modellnutzers von seinem Kunden eingenommen werden. Betrachtungsgegenstand sind also ausschließlich Prozesserhebungen, die durch menschliche Akteure durchgeführt werden. Nicht im Fokus des Beitrags stehen hingegen automatisierte Prozesserhebungsmethoden, die in das Unternehmen eingebettete Softwaresysteme voraussetzen. Eine sehr ausführliche Betrachtung solcher automatisierter Prozesserhebungsmethoden findet sich beispielsweise bei van der Aalst et al. (2003) oder bei Tiwari et al. (2008).

\section{Analyse ausgesuchter Literatur im Forschungsfeld}

Der vorliegende Beitrag erhebt nicht den Anspruch, den State-of-the-Art an Vorgehensmodellen vollumfänglich darzustellen, sondern beschränkt sich auf eine 
Auswahl etablierter deutschsprachiger Lehrbücher zum Geschäftsprozessmanagement, die auch die Zielgruppe der Praktiker und Unternehmensberater adressieren. Diese Quellenauswahl ist insofern angemessen, als dass sie einen guten Querschnitt der universitären Lehre widerspiegelt. Sollten diese Quellen keine operationalisierbaren Vorgehensmodelle zur Prozesserhebung bereitstellen, lässt dies den Schluss zu, dass in der universitären Ausbildung kein Standardvorgehen etabliert ist, das von der Beratungspraxis aufgegriffen werden kann.

Tabelle 1: Untersuchte Literaturquellen

\begin{tabular}{|c|l|c|l|}
\hline 1 & $\begin{array}{l}\text { Scheer (2002): ARIS - Vom Geschäfts- } \\
\text { prozess zum Anwendungssystem }\end{array}$ & 2 & $\begin{array}{l}\text { Allweyer (2005): Geschäftsprozess- } \\
\text { management }\end{array}$ \\
\hline 3 & $\begin{array}{l}\text { Becker/Kugeler/Rosemann (Hrsg.) } \\
\text { (2005): Prozessmanagement }\end{array}$ & 4 & $\begin{array}{l}\text { Fischer/Fleischmann/Obermeier } \\
\text { (2006): Geschäftsprozesse realisieren }\end{array}$ \\
\hline 5 & $\begin{array}{l}\text { Gaitanides (2007): } \\
\text { Prozessorganisation }\end{array}$ & 6 & $\begin{array}{l}\text { Fischermanns (2008): Praxishand- } \\
\text { buch Prozessmanagement }\end{array}$ \\
\hline 7 & $\begin{array}{l}\text { Gadatsch (2008): Grundkurs } \\
\text { Geschäftsprozessmanagement }\end{array}$ & \\
\hline
\end{tabular}

Tabelle 1 zeigt die sieben ausgewählten Standardlehrbücher zum Geschäftsprozessmanagement, die im Folgenden kritisch gewürdigt und in Tabelle 2 auf Seite 8 noch einmal zusammengefasst werden. Der Forschungsfrage entsprechend werden bei der Untersuchung v. a. die dort genannten Vorgehensmodelle zur Prozesserhebung sowie etwaige Hinweise zu ihrer Operationalisierung fokussiert.

1.) Bei Scheer werden inhaltlich zwei zentrale Themen behandelt, nämlich die Entwicklung des sog. ARIS-Konzeptes zur Beschreibung von Geschäftsprozessen sowie die Darstellung des ARIS House of Business Engineering (HOBE) als Modell zum Geschäftsprozessmanagement (Scheer 2002, Vorwort). Zum ARISKonzept wird ein grobes Vorgehensmodell vorgestellt und als Ereignisgesteuerte Prozesskette (EPK) abgebildet. Das Buch schließt mit Vorschlägen zum praktischen ARIS-Einsatz, die von drei Mitautoren mit Praxiserfahrung im jeweils behandelten Geschäftsfeld beigesteuert werden (Heib 2002, S. 147-153; Helling 2002, S. 154-161; Allweyer 2002, S. 162-168). Die beiden Vorgehensmodelle zum ARISKonzept und zum HOBE beschäftigen sich eher mit der allgemeinen Beschreibung von Inhalten der einzelnen Phasen als mit konkreten Vorschlägen zur ihrer Operationalisierung. Lediglich auf die Dokumentation von Geschäftsprozessen mittels unterschiedlichen Sichten wird ausführlich eingegangen. Hinweise, wie man diese Prozesse beispielsweise im Rahmen eines Workshops oder Interviews systematisch erheben könnte, werden nicht gegeben.

2.) Allweyer gliedert das Thema des Geschäftsprozessmanagements zunächst in einen Kreislauf aus vier Blöcken. Anschließend geht er auf das Thema Vorgehensmodelle ein, die er als nützliche Hilfsmittel zur Durchführung von Projekten charakterisiert und sie damit gleichzeitig vom zuvor dargestellten Kreislauf des 
Geschäftsprozessmanagements abgrenzt, der nach seinem Verständnis kontinuierlich abläuft und immer wieder von neuem beginnt (Allweyer 2005, S. 89-101). Trotz der ausführlichen und praxisorientierten Darstellung bleiben jedoch wichtige Themen der Prozesserhebung unberücksichtigt. So werden zwar Möglichkeiten zur Ist-Erhebung genannt, z. B. Workshops und Interviews, Beobachtung und Protokollierung von Abläufen oder Auswertung vorhandener Dokumente (Allweyer 2005, S. 224), Details zur Durchführung werden jedoch nicht thematisiert.

3.) Becker, Kugeler und Rosemann sowie ihre Mitautoren wollen mit ihrem Werk die Lücke zwischen theoretisch-konzeptionellen Werken zur Prozessorganisation und der Umsetzung dieser Konzepte in der Praxis schließen (Becker und Kahn 2005, S. 5-6). Dementsprechend stellen sie in Kapitel 2 ein Vorgehensmodell für die prozessorientierte Organisationsgestaltung vor, das sechs getrennte Phasen sowie ein phasenübergreifendes Projektmanagement umfasst. Nach Abschluss eines derart gestalteten Projektes zur Organisationsgestaltung schlagen die Autoren vor, ein kontinuierliches Prozessmanagement zur Sicherung der Wettbewerbsposition zu etablieren (Becker et al. 2005, S. 21-22). In der Phase Vorbereitung der Prozessmodellierung gehen die Autoren auf die Qualität von Informationsmodellen näher ein und erläutern die Grundsätze ordnungsmäßiger Modellierung (Rosemann et al. 2005, S. 46-49). Obschon diese Grundsätze näher beschrieben werden, erfolgt eine Konkretisierung jedoch nur im Hinblick auf Modellierungstechniken und Modellierungskonventionen. In der Phase Istmodellierung und Istanalyse legen die Autoren dar, dass vor der eigentlichen Istmodellierung eine Vorerfassung der Prozesse wichtig ist, und geben auch eine Liste von Merkmalen an, die im Vorfeld zu jedem Prozess erhoben werden sollten (Schwegmann und Laske 2005, S. 162-163). Offen bleibt jedoch, wie der Modellersteller an diese Informationen gelangen kann. Zur Erhebung und Dokumentation der Istmodelle werden Workshops vorgeschlagen und Aspekte genannt, die in diesen Workshops zu berücksichtigen sind (Schwegmann und Laske 2005, S. 165-167). Ohne weiteres ist das so skizzierte Vorgehen zur Prozesserhebung jedoch nicht operationalisierbar.

4.) Fischer, Fleischmann und Obermeier schlagen ein Vorgehensmodell mit fünf Ebenen zur Realisierung von Geschäftsprozessen vor, das sich ihnen zufolge in vielen Projekten zum Thema Prozessberatung bewährt hat (Fischer et al. 2006, S. 17). Das letzte Kapitel des Buches bietet Checklisten, die „als Gedankenhinweise und Arbeitsanleitungen für die praktische Arbeit" (Fischer et al. 2006, S. 161) dienen sollen. Diese Checklisten enthalten auch einen eigenen Abschnitt zum Thema der Prozessaufnahme, den die vorherigen Ausführungen zu den Ebenen des Vorgehensmodells thematisch jedoch nicht widerspiegeln. Hinweise zur Operationalisierung der in dieser Checkliste aufgeführten Maßnahmen zur Prozessaufnahme werden durch die Autoren nicht gegeben.

5.) Das Werk von Gaitanides hebt sich inhaltlich deutlich von den übrigen Literaturquellen ab. Bereits im Vorwort verdeutlicht der Autor, dass er die wissenschaftliche Diskussion des Prozessmanagements stärker in den Vordergrund rücken möchte, um durch eine theoretische Fundierung die praktische Arbeit zu 
unterstützen (Gaitanides 2007, Vorwort). Dementsprechend werden in Kapitel 3 zunächst vier Phasen des Reengineerings von Geschäftsprozessen eingeführt sowie Arbeiten untersucht, die sich mit diesen Phasen beschäftigen. Die erste Phase der Prozessidentifikation sieht der Autor als erfolgsbestimmenden Faktor, da durch sie alle weiteren Aktivitäten in einem Reengineering-Projekt determiniert werden. Seiner Meinung nach fokussiert die Praxis jedoch eher die dritte Phase, das Prozessdesign, in der Unternehmensberater überwiegend aktiv werden. Die Empfehlungen der Berater stellt er zwar nicht grundsätzlich in Frage, deklariert sie jedoch als völlig ungeeignet, um konkrete Veränderungen bzw. Verbesserungen anzuleiten (Gaitanides 2007, S. 57-58) und begründet so ein bestehendes Erkenntnisdefizit. Entsprechend der thematischen Ausrichtung des Werkes geht Gaitanides nicht auf die Thematik bzw. spezifischen Problemstellungen der Operationalisierung ein, die bei Erhebung des Ist-Zustands von Geschäftsprozessen auftreten können.

6.) Fischermanns gliedert seine Arbeit thematisch in vier große Blöcke. Er empfiehlt, die Prozessidentifikation zunächst soll-orientiert zu beginnen und das daraus abgeleitete Prozessmodell mit dem Ist-Zustand auf Vollständigkeit und Realitätsbezug abzugleichen (Fischermanns 2008, S. 104-105). Dieser Abgleich impliziert, dass zumindest eine rudimentäre Abbildung des Ist-Zustandes existieren muss. Diesbezüglich schlägt der Autor vor, bereits im Unternehmen vorhandene Dokumente mit entsprechenden Inhalten zu untersuchen sowie Workshops durchzuführen, um die Prozesse zunächst grob zu strukturieren und schließlich detailliert zu erheben. Die Dokumentation der Prozesse sollte dabei mit der im Unternehmen standardmäßig eingesetzten Modellierungstechnik erfolgen (Fischermanns 2008, S. 186). Von der Beschreibung einiger Modellierungstechniken sowie operationalisierbaren Angaben zur Erhebung von Zeitgerüsten abgesehen bleibt jedoch offen, wie in den Workshops konkret vorzugehen ist. Auch dem zuvor genannten Punkt der vorgelagerten Dokumentenanalyse wird unter Operationalisierungsgesichtspunkten keine nähere Beachtung geschenkt.

7.) Die letzte untersuchte Literaturquelle von Gadatsch bietet inhaltlich zunächst einen Abriss über die grundlegenden Begriffe des Prozessmanagements, des Business Reengineering sowie des Workflow-Managements, bevor der Autor näher auf das Thema Prozessmodellierung eingeht. Ähnlich wie bei Scheer wird ausführlich auf die Modellierung der einzelnen Sichten des ARIS-Konzeptes eingegangen, wobei der Autor seine theoretischen Ausführungen mit Beispielen und zusätzlichen Informationen unterfüttert. So widmet sich ein Abschnitt in Kapitel 3 z. B. Modellierungsfehlern und Modellierungsproblemen, die bei Anwendung der EPKMethode auftreten können (Gadatsch 2008, S. 220-225). Nicht thematisiert werden von Gadatsch hingegen die konkreten Fragestellungen, die im Rahmen der Prozesserhebung auftreten können. Zwar wird erwähnt, dass im Rahmen von Interviews die Ist-Geschäftsprozesse zu erheben sind, und der Autor gibt im Anschluss daran auch einige grundlegende Hinweise zu Interviewprinzipien (Gadatsch 2008, S. 35-37), er lässt jedoch offen, wie genau diese Erhebung sinnvoll unterstützt bzw. strukturiert und operationalisiert werden kann. 


\section{Ein Ansatz zur Klassifikation der untersuchten Modelle}

Die Literaturanalyse zeigt, dass alle Autoren mindestens ein, z. T. aber auch mehrere Vorgehensmodelle zum Geschäftsprozessmanagement bzw. zur Geschäftsprozessreorganisation vorschlagen. Um diese unterschiedlichen Vorgehensmodelle zu klassifizieren, wird im Folgenden der in Abbildung 2 dargestellte morphologische Kasten verwendet, der sechs Merkmale mit ihren jeweils möglichen Ausprägungen umfasst. Die untersuchten Quellen werden mit der Nummerierung aus Tabelle 1 (Seite 4) in das Klassifikationsschema eingeordnet.

Zunächst werden die allgemeinen Charakteristika des jeweiligen Vorgehensmodells anhand dreier Merkmale verortet. Das erste Merkmal Modellkontext bestimmt, ob ein Vorgehensmodell eher auf eine einmaligen (Re-) Organisation der Geschäftsprozesse ausgelegt ist oder zum kontinuierlichen Management von Geschäftsprozessen herangezogen werden kann. Das zweite Merkmal bezieht sich auf die Art des Vorgehensmodells und unterscheidet zwischen sequentiellen, sequentiell/zyklischen und zyklischen Modellen. Bei zyklischen Modellen werden die einzelnen Phasen immer wieder in Form eines Kreislaufs durchlaufen, während bei sequentiellen Modellen die einzelnen Phasen nacheinander angeordnet sind. Die Mischform sequentiell/zyklisch bezeichnet Vorgehensmodelle, die zwar eigentlich eine sequentielle Phasenfolge aufweisen, aber mit einer letzten Phase abschließen, die das kontinuierliche Prozessmanagement zur Aufgabe hat. Das dritte Merkmal Phasen brw. Ebenen trägt dem Umstand Rechnung, dass die untersuchten Vorgehensmodelle in unterschiedliche Phasen gegliedert sind.

Die übrigen drei Merkmale fokussieren die Prozesserhebung im Besonderen. Die Literaturanalyse zeigt, dass die Autoren insgesamt fünf Methoden zur Informationsgewinnung nennen, die im Rahmen der Prozesserhebung eingesetzt werden können. Die häufigsten Nennungen entfielen dabei auf das Studium bereits vorhandener Dokumente sowie die Durchführung von Interviews mit Fachexperten. Hervorzuheben ist hier vor allem, dass in keiner der untersuchten Quellen detailliert dargelegt wird, wie genau diese Erhebung von Informationen zu Geschäftsprozessen bei Anwendung der verschiedenen Methoden durchgeführt werden kann bzw. durchgeführt werden sollte. Auf die Erhebung der Prozesse folgt ihre Dokumentation, die Gegenstand des fünften Merkmals ist, das die Frage beantwortet, mit welcher Modellierungsmethode bzw. Notation die erhobenen Geschäftsprozesse abgebildet werden können. 


\section{Tabelle 2: Übersicht über die untersuchten Literaturquellen}

\begin{tabular}{|c|c|c|c|}
\hline & 1) Scheer & 1) Heib in Scheer & 2) Allweyer \\
\hline $\begin{array}{l}\text { Vorgehensmodell } \\
(\mathrm{VM})\end{array}$ & $\begin{array}{l}\text { 1. Grobes ARIS-VM bzw. } \\
\text { 2. ARIS-HOBE }\end{array}$ & $\begin{array}{l}\text { VM zur } \\
\text { Geschäftsprozessoptimierung }\end{array}$ & Geschäftsprozess-Management Kreislauf \\
\hline \multirow{4}{*}{$\begin{array}{l}\text { Inhalte mit Bezug zur } \\
\text { Prozesserhebung }\end{array}$} & \multirow{2}{*}{$\begin{array}{l}\text { 1. Lediglich Aussage, dass aus } \\
\text { allgemeinem Modell projekt- } \\
\text { spezifische VM abgeleitet werden } \\
\text { können }\end{array}$} & $\begin{array}{l}\text { Definition Grobziele; Projektteam } \\
\text { festlegen; Konventionen- und } \\
\text { Projekthandbuch erstellen }\end{array}$ & $\begin{array}{l}\text { Anpassung Geschäftsprozesse an die } \\
\text { Unternehmensstrategie; Kernprozesse } \\
\text { festlegen }\end{array}$ \\
\hline & & $\begin{array}{l}\text { Dokumentation von Produkt- und } \\
\text { Leistungsmodellen; Konretisierung } \\
\text { der Projektziele }\end{array}$ & \multirow{2}{*}{$\begin{array}{l}\text { ARIS als Rahmenkonzept; EPK; } \\
\text { Geschäftsregeln; Objektorientierte } \\
\text { Notationen; Modellierungswerkzeuge und } \\
\text {-konventionen }\end{array}$} \\
\hline & \multirow{2}{*}{$\begin{array}{l}\text { 2. Strategische Unternehmens- } \\
\text { planung als Ausgangspunkt der } \\
\text { Geschäftsprozessgestaltung; } \\
\text { Anpassung von Referenz- } \\
\text { modellen; Prozessbewertung; } \\
\text { Benchmarking }\end{array}$} & \multirow[b]{2}{*}{$\begin{array}{l}\text { Bestandsaufnahme Geschäfts- } \\
\text { prozesse; WSK-Diagramme; EPK- } \\
\text { Methode; Identifikation von } \\
\text { Schwachstellen/Verbesserungs- } \\
\text { potenzialen }\end{array}$} & \\
\hline & & & $\begin{array}{l}\text { Methoden Ist-Erhebung inkl. autom. } \\
\text { Lösungen; Vor- und Nachteile Ist- } \\
\text { Analyse; Kriterien für die Analyse; } \\
\text { Kennzahlenermittlung; Benchmarking }\end{array}$ \\
\hline $\begin{array}{l}\text { Aussagen zur } \\
\text { Operationalisierung }\end{array}$ & \multicolumn{2}{|l|}{ 3) Becker/Kugeler/Rosemann } & 4) Fischer/Fleischmann/Obermeier \\
\hline $\begin{array}{l}\text { Vorgehensmodell } \\
(\mathrm{VM})\end{array}$ & \multicolumn{2}{|c|}{ Vorgehen eines prozessorientierten Reorganisationsprojekts } & $\begin{array}{l}\text { Fünf-Schichten-Modell zur Realisierung } \\
\text { von Geschäftsprozessen }\end{array}$ \\
\hline \multirow{4}{*}{$\begin{array}{l}\text { Inhalte mit Bezug zur } \\
\text { Prozesserhebung }\end{array}$} & \multirow{2}{*}{\multicolumn{2}{|c|}{$\begin{array}{l}\text { Grundsätze ordnungsgem. Modellierung; Einsatzzwecke Prozessmodelle; } \\
\text { Auswahl Modelltypen; Modellierungskonventionen und -werkzeuge; } \\
\text { Verwendung Modellierungsstandard }\end{array}$}} & Differenzierung der Prozesstypen \\
\hline & & & $\begin{array}{l}\text { Strukturierbarkeit von und Sichten auf } \\
\text { Geschäftsprozesse }\end{array}$ \\
\hline & \multicolumn{2}{|c|}{ Struktur- und Prozessziele festlegen; Kernprozesse identifizieren } & \multirow[b]{2}{*}{$\begin{array}{l}\text { Kriterien zur Prozessbeschreibung; Arten } \\
\text { von Prozessbeschreibungen (textuell- } \\
\text { informell, flussorientiert, objektorientiert, } \\
\text { subjektorientiert) }\end{array}$} \\
\hline & \multicolumn{2}{|c|}{$\begin{array}{l}\text { Argumente pro/contra Istmodellierung; Identifikation Informations- } \\
\text { quellen; Identifikation zu erhebende Prozesse; grobe Erfassung der } \\
\text { Abläufe; Erstellung Istmodelle (Workshops); Identifikation von } \\
\text { Schwachstellen/Verbesserungspotenzialen; Modellkonsolidierung }\end{array}$} & \\
\hline $\begin{array}{l}\text { Aussagen zur } \\
\text { Operationalisierung }\end{array}$ & 5) Gaitanides & 6) Fischermanns & 7) Gadatsch \\
\hline $\begin{array}{l}\text { Vorgehensmodell } \\
(\mathrm{VM})\end{array}$ & $\begin{array}{l}\text { Phasen des Reengineering von } \\
\text { Geschäftsprozessen }\end{array}$ & ibo-Prozessfenster & $\begin{array}{l}\text { Integriertes Geschäftsprozess- und } \\
\text { Workflow-Management }\end{array}$ \\
\hline \multirow{4}{*}{$\begin{array}{l}\text { Inhalte mit Bezug zur } \\
\text { Prozesserhebung }\end{array}$} & \multirow{2}{*}{$\begin{array}{l}\text { Deduktiver vs. Induktiver } \\
\text { Prozessentwurf; Markt- vs. } \\
\text { Ressourcenorientierter } \\
\text { Prozessentwurf }\end{array}$} & \multirow{3}{*}{$\begin{array}{l}\text { Prozessidentifikation; Aufbau } \\
\text { Unternehmensprozessmodell } \\
\text { (oberste Gliederungsebene); } \\
\text { Festlegung Prozessziele; } \\
\text { Dokumentenstudium (vorhandene } \\
\text { Prozessmodelle); Erstellung von } \\
\text { Prozessskizzen (Workshops); } \\
\text { Identifikation Kernprozesse }\end{array}$} & $\begin{array}{l}\text { Auswahl der zu modellierenden und zu } \\
\text { implementierenden Prozesse }\end{array}$ \\
\hline & & & $\begin{array}{l}\text { Anwendung Sichtenkonzept zur } \\
\text { Komplexitätsreduktion; Methoden der }\end{array}$ \\
\hline & \multirow{2}{*}{$\begin{array}{l}\text { Zwecke Prozessmodellierung; } \\
\text { Gliederungskriterien zur } \\
\text { Strukturierung/Konstruktion von } \\
\text { Geschäftsprozessen; } \\
\text { Detaillierungsgrad der } \\
\text { Gesamtarchitektur; Software- } \\
\text { Tools zur Unterstützung der } \\
\text { Prozessmodellierung }\end{array}$} & & $\begin{array}{l}\text { Prozessmodellierung (Datenfluss-, } \\
\text { Kontrollfluss-, Objektorientiert); } \\
\text { Werkzeuge Prozessmanagement; ARIS- } \\
\text { Konzept }\end{array}$ \\
\hline & & $\begin{array}{l}\text { Aufnahme Ist-Prozesse; Prozess- } \\
\text { erhebung (Workshops); Erläuterung } \\
\text { Laufzettelverfahren; Prozess- } \\
\text { modellierung; Prozessanalyse }\end{array}$ & $\begin{array}{l}\text { Ableitung Führungsgrößen aus strateg. } \\
\text { Geschäftsfeldern; Ermittlung } \\
\text { Erfolgsdefizite/Aufdeckung von } \\
\text { Schwachstellen }\end{array}$ \\
\hline $\begin{array}{l}\text { Aussagen zur } \\
\text { Operationalisierung }\end{array}$ & $\begin{array}{l}\text { 冈 Autor geht nicht auf } \\
\text { Thematik/spezifische Problem- } \\
\text { stellungen ein, die bei Erhebung } \\
\text { des Ist-Zustandes von } \\
\text { Geschäftsprozessen auftreten }\end{array}$ & $\begin{array}{l}\text { ■ Operationalisierbare Angaben zur } \\
\text { Erhebung von Zeitgerüsten } \\
\text { 囚 Keine Hinweise, wie Prozess- } \\
\text { erhebung bei Dokumentenstudium } \\
\text { /in Workshops erfolgen sollte }\end{array}$ & $\begin{array}{l}\text { च Ausführliche Darstellung Model- } \\
\text { lierungsmethoden/ARIS-Konzept } \\
\text { 冈 Keine Hinweise, wie bei der } \\
\text { Prozesserhebung mittels Interviews } \\
\text { konkret vorgegangen werden sollte }\end{array}$ \\
\hline
\end{tabular}


Im Hinblick auf die Operationalisierbarkeit gibt die jeweilige Notation Regeln und damit Einschränkungen für den Modellkonstrukteur vor. In den untersuchten Quellen werden zahlreiche mögliche Ausprägungen erläutert, die bei vollständiger Erfassung den Rahmen dieses Beitrags sprengen würden. Daher beschränkt sich die Darstellung innerhalb des morphologischen Kastens auf die häufigsten Vertreter.

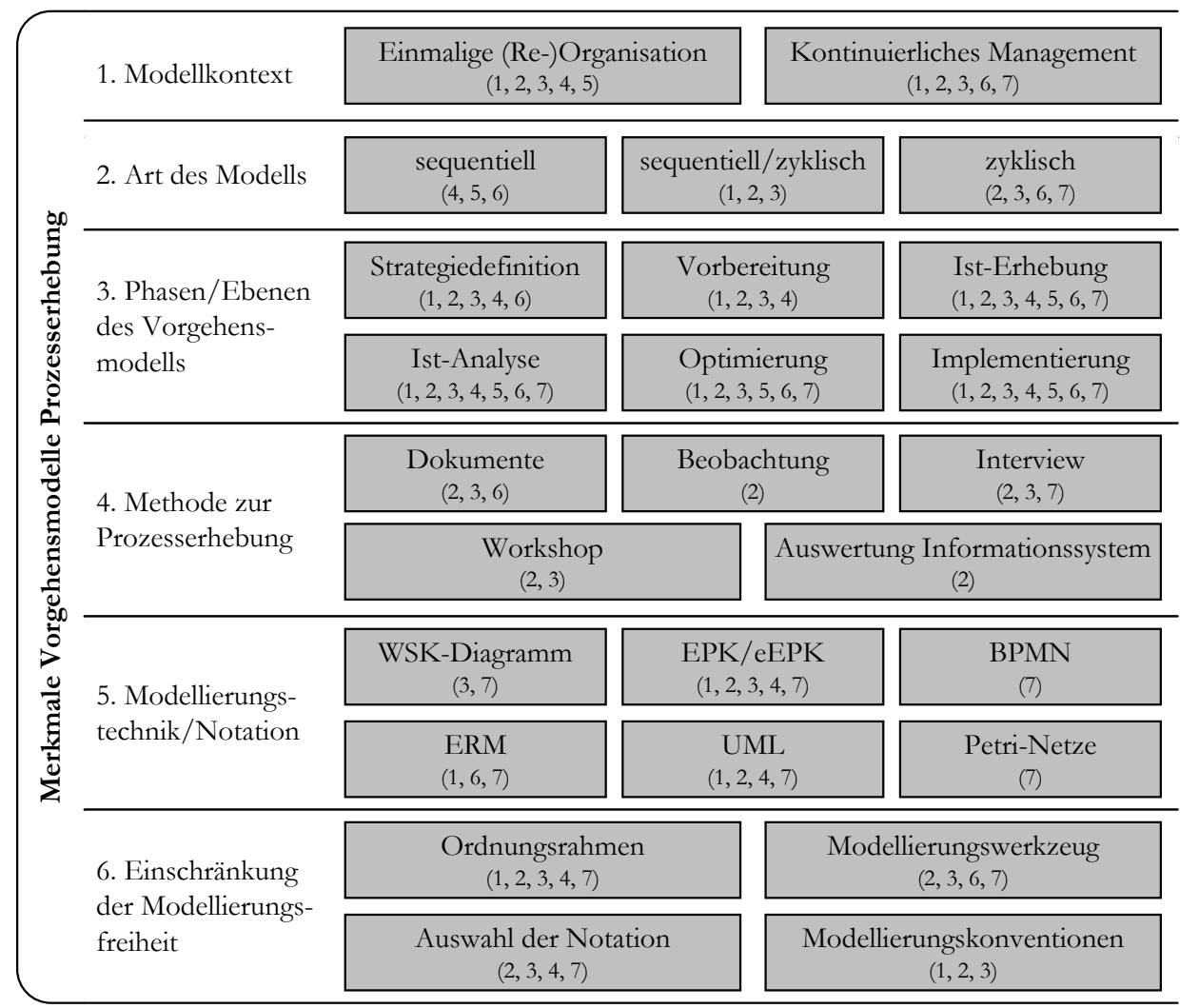

\section{Abbildung 2: Klassifikation geschäftsprozessorientierter Vorgehensmodelle}

Die sechste und letzte Dimension dient der Einordnung von Methoden, die den Modellkonstrukteur in seiner Modellierungsfreibeit einscbränken. Dieser Aspekt ist für den vorliegenden Beitrag von Bedeutung, da unter Operationalisierungsgesichtspunkten auch zu untersuchen ist, wie der Modellersteller eigentlich von den erhobenen Informationen $\mathrm{zu}$ den jeweiligen Geschäftsprozessmodellen kommt. Diese Frage wird in den untersuchten Quellen weitgehend ausgeklammert, indem der Vorgang als kreativer Prozess deklariert wird, der sich gewissermaßen „im Kopf des Modellerstellers“ abspielt. Dieser kreative Prozess wird jedoch beispielsweise durch die Einhaltung eines Ordnungsrahmens, die Auswabl eines Modellie- 
rungswerkzengs, die gewäblte Notation oder das Einhalten von Modellierungskonventionen begrenzt. Dementsprechend werden diese Mittel zur Einschränkung der Modellierungsfreiheit auch in den untersuchten Quellen mehr oder weniger detailliert behandelt und können damit zur Klassifikation herangezogen werden.

\section{Ergebnisse und Implikationen für die Beratungspraxis}

Im vorliegenden Beitrag wurden sieben Literaturquellen auf Vorgehensmodelle zum Geschäftsprozessmanagement im Hinblick auf die Operationalisierung der Geschäftsprozesserhebung untersucht. Daraus wurde ein Ansatz zur Klassifikation der untersuchten Vorgehensmodelle abgeleitet, in dem die jeweiligen thematischen Schwerpunkte der untersuchten Quellen verortet werden können.

Insgesamt bietet die untersuchte Literatur kaum konkrete Ansatzpunkte zur Umsetzung bzw. Durchführung einer Geschäftsprozesserhebung. Lediglich Modellierungsnotationen und Methoden, die die Modellierungsfreiheit einschränken, werden z. T. sehr detailliert behandelt. Die Informationsgewinnung, z. B. durch Dokumentenstudium bzw. Interviews oder Workshops mit Fachanwendern, wird zwar kurz angesprochen, konkrete Handlungsempfehlungen zum operativen Vorgehen oder zur Problembehandlung werden jedoch nicht gegeben. Ebenso wird das Thema der intersubjektiven Modellbildung zwischen Modellersteller und Modellnutzer kaum thematisiert - vereinzelt wird die fehlende Operationalisierung damit begründet, dass es sich um kreative Prozesse handele, die sich der Formalisierung entzögen.

Für die Beratungspraxis implizieren diese Ergebnisse, dass zurzeit keine einheitlichen, theoretisch fundierten Handlungsempfehlungen zur durchgängigen Unterstützung aller Tätigkeiten im Rahmen der Geschäftsprozesserhebung vorliegen. Vor dem Hintergrund der Wertschöpfungspotenziale in der Prozessberatung ist diese Erkenntnis paradox, da Beratungskunden i. A. sehr hohe Erwartungen an die Methodenkompetenz eines Beraters stellen, die dadurch ein zentrales Kriterium zur Abgrenzung von Wettbewerben wird (Hess und Schuller 2005, S. 371). Zwar steht zu vermuten, dass etablierte Beratungsunternehmen entsprechende Vorgehensmodelle entwickelt haben und einsetzen, sie diese jedoch als schutzwürdiges Wissen einstufen und daher nicht veröffentlichen. Einer wissenschaftlichen Diskussion der Prozesserhebung aus praktischer Sicht fehlt damit die Grundlage, da auch die durchgeführte Literaturanalyse zum Geschäftsprozessmanagement gezeigt hat, dass sich in der Wissenschaft bisher kein Standard für entsprechende Vorgehensmodelle herausgebildet hat und über die Art und Weise, wie eine Geschäftsprozesserhebung durchzuführen ist, keine Einigkeit besteht.

Gleichwohl könnte die Beratungspraxis von einer Öffnung hin zur Wissenschaft profitieren. Das Risiko, die eigenen Vorgehensweisen transparent zu machen, ist z. B. schon durch Personalfluktuation gegeben, da ausscheidende Mitarbeiter das hausinterne Methodenwissen zwangsläufig mit zum neuen Arbeitgeber 
nehmen. Würden solche Vorgehensmodelle jedoch regulär in den wissenschaftlichen Diskurs eingebracht, würde mit ihrer Verbreitung auch ihre qualitative Verbesserung durch Feedback aus Forschung und Lehre einher gehen. Darüber hinaus könnte die universitäre Lehre den Beratungsunternehmen so einen Teil der Vermittlung des notwendigen Methodenwissens abnehmen, das sie ihren neuen Mitarbeitern bisher mit hohem Aufwand selbst beibringen muss.

\section{Ausblick}

Die in diesem Beitrag vorgestellten Ergebnisse basieren auf der Untersuchung einer limitierten Anzahl von etablierten Standardwerken zum Geschäftsprozessmanagement. Ziel der durchgeführten Untersuchung war es nicht, den vollständigen State-of-the-Art an Vorgehensmodellen in diesem Themengebiet darzustellen, sondern ein Bewusstsein dafür zu schaffen, dass in der Literatur ein Mangel an operationalisierbaren Vorgehensmodellen zur Geschäftsprozesserhebung besteht. Vor diesem Hintergrund erfüllt die ausgewählte Literatur ihren Zweck, auf das vermutete Erkenntnisdefizit aufmerksam zu machen. Gleichwohl würden die hier vorgestellten Ergebnisse durch ein breiter angelegtes Literaturreview weiter an Qualität gewinnen. Dabei sollten auch verwandte Ansätze, wie beispielsweise das von Krallmann et al. (2002) diskutierte Requirements Engineering, eingebettet werden, um Aussagen darüber treffen zu können, inwieweit die Arbeiten aus den verwandten Bereichen für das Geschäftsprozessmanagement fruchtbar gemacht werden können. Auch die Untersuchung von bestehenden Ansätzen in der Unternehmensberatungspraxis würde einen sinnvollen Beitrag zu weiteren Forschungsbemühungen darstellen.

Der in Abschnitt 4 vorgestellte morphologische Kasten ist lediglich als erster Systematisierungsvorschlag für die untersuchten Vorgehensmodelle zu verstehen und könnte durch weitere Untersuchungen ausdifferenziert werden. Darauf aufbauend wäre dann perspektivisch ein Vorgehensmodell mit konkreten Hinweisen zur operativen Umsetzung der einzelnen Modellphasen zu entwickeln, das in der Unternehmenspraxis eingesetzt und dadurch überprüft sowie verbessert werden könnte.

\section{Literatur}

Allweyer T (2005) Geschäftsprozessmanagement. Strategie, Entwurf, Implementierung, Controlling. W3L GmbH, Herdecke.

Becker J, Berning W, Kahn D (2005) Projektmanagement. In: Becker J, Kugeler M, Rosemann M (Hrsg.) Prozessmanagement - Ein Leitfaden zur prozessorientierten Organisationsgestaltung. Springer, Berlin. 
Becker J, Kahn D (2005) Der Prozess im Fokus. In: Becker J, Kugeler M, Rosemann M (Hrsg.) Prozessmanagement - Ein Leitfaden zur prozessorientierten Organisationsgestaltung. Springer, Berlin.

Bund Deutscher Unternehmensberater e.V. (2009) Der Markt für Managementberatung. http://www.bdu.de/docs/downloads/BDU_Online/

Branche/Markt\%20Managementberatung\%202008-2009.pdf. Abruf am 200909-12.

Eggert S (2009) Das aktuelle Stichwort: Prozessmanagement. ERP Management $5: 16$.

Fischer H, Fleischmann A, Obermeier S (2006) Geschäftsprozesse realisieren. Ein praxisorientierter Leitfaden von der Strategie bis zur Implementierung. Vieweg, Wiesbaden.

Fischermanns G (2008) Praxishandbuch Prozessmanagement. Verlag Dr. Götz Schmidt, Gießen.

Gadatsch A (2008) Grundkurs Geschäftsprozessmanagement. Methoden und Werkzeuge für die IT-Praxis: Eine Einführung für Studenten und Praktiker. Vieweg, Wiesbaden.

Gaitanides M (2007) Prozessorganisation. Entwicklung, Ansätze und Programme des Managements von Geschäftsprozessen. Vahlen, München.

Hammer M, Champy J (1994) Business Re-Engineering. Campus, Frankfurt.

Hess T, Schuller D (2005) Business Process Reengineering als nachhaltiger Trend? Eine Analyse der Praxis in deutschen Großunternehmen nach einer Dekade. ZFBF 57:355-373.

Jost W, Kruppke H (2004) Business Process Management: der ARIS Value Engineering-Ansatz. In: Scheer AW, Abolhassan F, Kruppke H, Jost W (Hrsg.) Innovation durch Geschäftsprozessmanagement, Jahrbuch Business Process Excellence 2004/2005. Springer, Berlin.

Krallmann H, Frank H, Gronau N (Hrsg.) (2002) Systemanalyse im Unternehmen: Vorgehensmodelle, Modellierungsverfahren und Gestaltungsoptionen. Oldenbourg, Berlin.

Nissen V (2007) Consulting Research - Eine Einführung. In: Nissen V (Hrsg) Consulting Research - Unternehmensberatung aus wissenschaftlicher Perspektive. Gabler, Wiesbaden.

Rosemann M, Schwegmann A, Delfmann P (2005) Vorbereitung der Prozessmodellierung. In: Becker J, Kugeler M, Rosemann M (Hrsg.) Prozessmanagement - Ein Leitfaden zur prozessorientierten Organisationsgestaltung. Springer, Berlin. 
Scheer AW (2002) ARIS - Vom Geschäftsprozess zum Anwendungssystem. Springer, Berlin.

Schütte R (1998) Grundsätze ordnungsmäßiger Referenzmodellierung - Konstruktion konfigurations- und anpassungsorientierter Modelle. Gabler, Wiesbaden.

Schwegmann A, Laske M (2005) Istmodellierung und Istanalyse. In: Becker J, Kugeler M, Rosemann M (Hrsg.) Prozessmanagement - Ein Leitfaden zur prozessorientierten Organisationsgestaltung. Springer, Berlin.

Tiwari A, Turner CJ, Majeed B (2008) A review of business process mining: stateof-the-art and future trends. Business Process Management Journal 14(1): 5-22.

Van der Aalst WMP, van Dogen BF, Herbst J, Maruster L, Schimm G, Weijters AJMM (2003) Workflow mining: A survey of issues and approaches. Data \& Knowledge Engineering 47: 237-267.

Vom Brocke J (2003) Referenzmodellierung - Gestaltung und Verteilung von Konstruktionsbegriffen. Logos, Berlin. 